\title{
High Real-Space Resolution Structure of Materials by High-Energy X-Ray Diffraction
}

\author{
V. Petkov, ' S.J.L. Billinge, ${ }^{1}$ J. Heising, ${ }^{2}$ M. G. Kanatzidis, ${ }^{2}$ S. Shastri, ${ }^{3}$ and S. Kycia ${ }^{4}$ \\ ${ }^{1}$ Department of Physics and Astronomy and Center for Fundamental Materials Research, Michigan \\ State University, East Lansing, MI 48823 \\ ${ }^{1}$ Department of Chemistry and Center for Fundamental Materials Research, Michigan State \\ University, East Lansing, MI 48823 \\ ${ }^{3}$ Advanced Photon Source, Argonne National Laboratory,* Argonne, IL 60439, USA \\ ${ }^{4}$ Cornell High Energy Synchrotron Source, Cornell University, Ithaca, NY 14853
}

November 1999

The submitted manuscript has been created by
the University of Chicago as Operator of
Argonne National Laboratory ("Argonne") under
Contract No. W-31-109-ENG-38 with the U.S.
Department of Energy. The U.S. Government
retains for itself, and others acting on its behalf,
a paid-up, nonexclusive, irrevocable worldwide
license in said article to reproduce, prepare
derivative works, distribute copies to the public,
and perform publicly and display publicly, by
or on behalf of the Government.

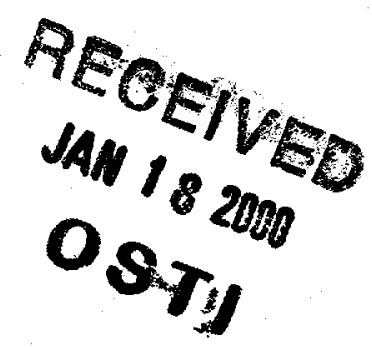

To be presented at the Fall'99 MRS Meeting, Boston, MA, Nov 29-Dec 3, 1999; to be published in the Proceedings.

\footnotetext{
*This work is supported by the U.S. Department of Energy, Basic Energy Sciences, Office of Science, under contract \#W-31-109-ENG-38.
} 


\section{DISCLAIMER}

This report was prepared as an account of work sponsored by an agency of the United States Government. Neither the United States Government nor any agency thereof, nor any of their employees, make any warranty, express or implied, or assumes any legal liability or responsibility for the accuracy, completeness, or usefulness of any information, apparatus, product, or process disclosed, or represents that its use would not infringe privately owned rights. Reference herein to any specific commercial product, process, or service by trade name, trademark, manufacturer, or otherwise does not necessarily constitute or imply its endorsement, recommendation, or favoring by the United States Government or any agency thereof. The views and opinions of authors expressed herein do not necessarily state or reflect those of the United States Government or any agency thereof. 


\section{DISCLAIMER}

Portions of this document may be illegible in electronic image products. Images are produced from the best available original document. 


\title{
High real-space resolution structure of materials by high-energy $\mathrm{x}$-ray diffraction
}

\author{
V. PETKOV' ${ }^{\prime}$, S. J.L. BILLINGE', J. HEISING ${ }^{2}$, M. G. KANATZIDIS ${ }^{2}$, S. SHASTRI ${ }^{3}$, \\ and S. KYCIA ${ }^{+}$ \\ 'Department of Physics and Astronomy and Center for Fundamental Materials Research, \\ Michigan State University, East Lansing, MI 48823 \\ ${ }^{2}$ Department of Chemistry and Center for Fundamental Materials Research, Michigan \\ State University, East Lansing, MI 48823 \\ ${ }^{3}$ Advanced Photon Source, Argonne National Laboratory, Argonne, IL 60439 \\ ${ }^{4}$ Cornell High Energy Synchrotron Source, Cornell University, Ithaka, NY 14853
}

\begin{abstract}
Results of high-energy synchrotron radiation experiments are presented demonstrating the advantages of the atomic Pair Distribution Function technique in determining the structure of materials with high resolution.
\end{abstract}

\section{INTRODUCTION}

It is well known that physical properties and technological characteristics of materials are, to a great extent, predetermined by the atomic-scale structure. Also, most of technologically important materials are not mono but polycrystalline in their nature. That is why a lot of effort has been exercised to develop techniques for determining the structure of polycrystalline materials. Real progress has been made in the field by employing the so-called Rietveld technique [1]. Essentially it is a least-squares refinement of crystal structure parameters, specimen characteristics, diffraction optics and instrumental factors carried out until the best possible agreement between the observed and calculated powder diffraction patterns is obtained. A peculiarity of the technique is that it relies on the Bragg intensities only while the rest of the diffraction pattern is considered to be an uninformative background contribution. Nowadays the structure of polycrystalline materials of variable structural complexity, ranging from disperse catalysts to ceramic high-Tc semiconductors and even simple organic macromolecules is being almost routinely refined by the Rietveld technique [2]. However, increasingly many new interesting materials contain significant disorder on an atomic scale. Often this disorder has a direct effect on the properties which make the material technologically and/or scientifically important. It is clearly necessary to have a technique which can characterize not only the average, long-range structure but the deviation from it, i.e. the local disorder as well. Information about the local structural disorder is, however, contained in the diffuse scattering which is of low intensity and is usually widely spread in reciprocal space. A fruitful experimental approach which can handle both Bragg intensities and the diffuse component is the so-called atomic Pair Distribution Function (PDF) technique. With the PDF technique both Bragg intensities and the diffuse component(s) of the total diffraction spectrum are treated simultaneously and then Fourier transformed to yield the atomic PDF which is thus a representation of both the long- 
range (average) and short-range (local) atomic structure of the material. Since the PDF is obtained with no assumption of periodicity, glassy materials as well as polycrystals exhibiting a different degree of local disorder can be characterized employing the same approach [3]. To apply the technique usefully the PDF has to be of high resolution. To do this, accurate total scattering intensity has to be measured over a wide range of diffraction vectors, $Q$. It implies collecting data with $\mathrm{x}$-rays of high incident energies, i.e. the use of synchrotron sources of $\mathrm{x}$ rays. In the present paper selected examples of such high-resolution PDF studies are presented.

\section{FUNDAMENTALS OF THE PDF TECHNIQUE}

The atomic PDF, G(r), is defined as follows:

$$
G(r)=4 \pi r\left[\rho(r)-\rho_{o}\right]
$$

where $\rho(r)$ and $\rho_{o}$ are the local and average atomic number densities, respectively and $r$ is the radial distance. $G(r)$ is a measure of the probability of finding an atom at a distance $r$ from a reference atom and so describes the atomic arrangement, i.e. structure, of materials. It is the sine Fourier transform of the experimentally observable total structure function, $S(Q)$, i.e.

$$
G(r)=(2 / \pi) \int_{Q=o}^{Q_{\max }} Q[S(Q)-1] \sin (Q r) d Q
$$

where $Q$ is the magnitude of the wave vector. The structure function is related to only the coherent part of the total diffraction spectrum of the material as follows:

$$
S(Q)=1+\left[I^{\cos }(Q)-\sum c_{i}\left|f_{i}(Q)\right|^{2}\right] /\left|\sum c_{i} f_{i}(Q)\right|^{2}
$$

where $\Gamma^{o h} .(Q)$ is the coherent scattering intensity per atom in electron units and $c_{\mathrm{i}}$ and $f_{i}$ are the atomic concentration and scattering factor for the atomic species of type $i$, respectively [4]. The following important details of the PDF technique are to be noted: $G(r)$ is barely influenced by diffraction optics and experimental factors since these are accounted for in the step of extracting the coherent intensities from the raw diffraction data. In the present studies it was done with the help of the program RAD [5]. As eq. 2 implies all but not only Bragg diffracted intensities contribute to $G(r)$. Also, by accessing high values of $Q$, experimental $G(r) s$ of high real-space resolution can be obtained and, hence, quite fine structural features revealed. The latest point is

well demonstrated by the present studies where wave vectors as high as $45 \AA^{-1}$ were achieved with the use of intense synchrotron sources of radiation. All these features make the PDF technique a natural approach when the real atomic, both long and short-range order, structure of materials is needed.

\section{RESULTS}

\section{A. Local atomic structure of $\operatorname{In}_{I_{-x}} G a_{x}$ As semiconductor alloys}

Ternary semiconductor alloys, in particular $\ln h_{x} G a_{x} A s$, have technological importance because they allow useful properties, such as band-gaps, to be varied continuously between the two end 


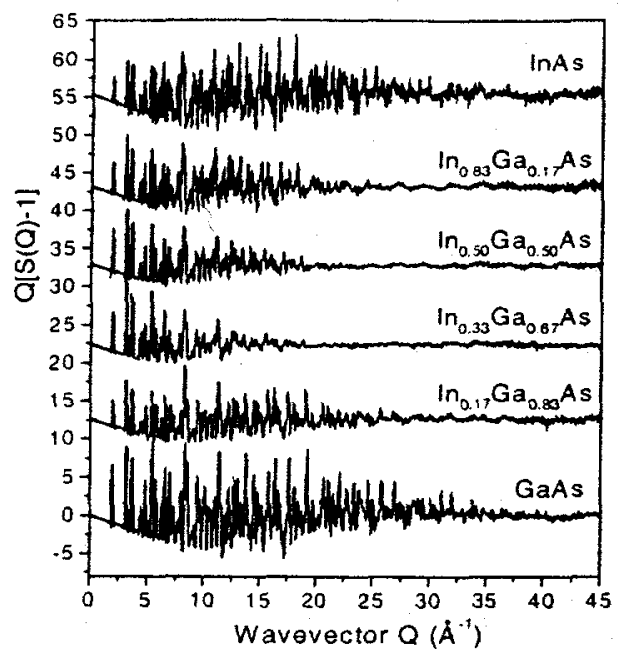

Figure 1. Experimental structure factors for $I n_{f-x} G a_{x} A s$ alloys.

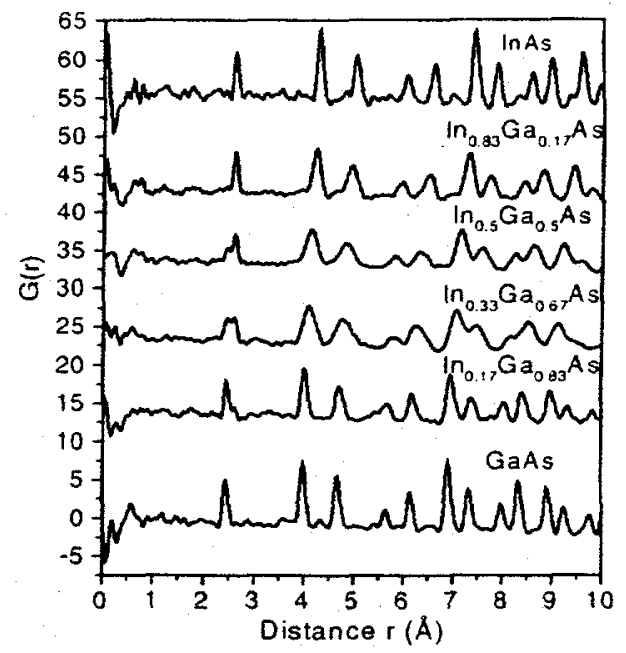

Figure 2. Reduced atomic PDFs for for $\ln _{l-x} G a_{x} A s$ alloys.

points by varying the composition, $x$. On average, $I n_{l-x} G a_{x} A s$ alloys are of the zinc-blende type structure where metal $(I n ; G a)$ and $A s$ atoms occupy two interpenetrating $f c c$ lattices. Due to the considerably different bond lengths present, $\mathrm{L}_{\mathrm{In}-\mathrm{As}}=2.61 \AA$ and $\mathrm{L}_{\mathrm{Ga}-\mathrm{As}}=2.437 \AA$, the zinc-blende lattice of the alloy is, however, locally distorted. The real, i.e. distorted, structure is a prerequisite to any accurate band-structure and phonon dispersion calculations and that is why we undertook a PDF study to determine it. We carried out diffraction experiments at the A2 24 pole wiggler beamline at CHESS using $x$-rays of energy $60 \mathrm{keV}$. More experimental details can be found in refs. [6,7]. Experimental reduced structure factors and the corresponding atomic PDFs $G(r)$ are shown in Figs. 1 and 2, respectively. Significant Bragg scattering (well-defined peaks) are seen with the end-members, InAs and GaAs, up to $35 \AA^{-1}$. This implies that the samples have longrange order and there is little positional disorder. The Bragg peaks disappear at much lower $Q$ values with the alloys: the samples are still long-range ordered but they have significant local positional disorder. From the second neighbour onwards this disorder results in broad atomicpair distributions as can be seen in Fig. 2. The nearest-neighbour peak is the only peak which remains sharp. In the alloy samples it is clearly split into a doublet with low and higher-r components corresponding to $G a-A s$ and $I n$-As bonds, respectively. A simple structure model based on the 8-atom cubic unit cell of $(I n ; G a)$ As has been fit to the experimental PDFs and the way the underlying zinc-blende structure of the alloys distorts locally to accommodate the bondlength mismatch has been quantified. It has been found that both metal $(\mathrm{In} ; G a)$ and $A$ s atoms are statically displaced from their positions in the ideal lattice. Extra positional disorder, manifested by enlarged temperature factors, has been found on both metal and As sites as well. Both the static displacement and the positional disorder have been found to peak at a composition $x=0.5$ $[6,7]$. 


\section{B. Average atomic structure of "restacked" WS}

Due to a unique combination of valuable structural, electronic and optical properties, the layered dichalcogenides, such as $W S_{2}$, have been studied and used for many practical applications

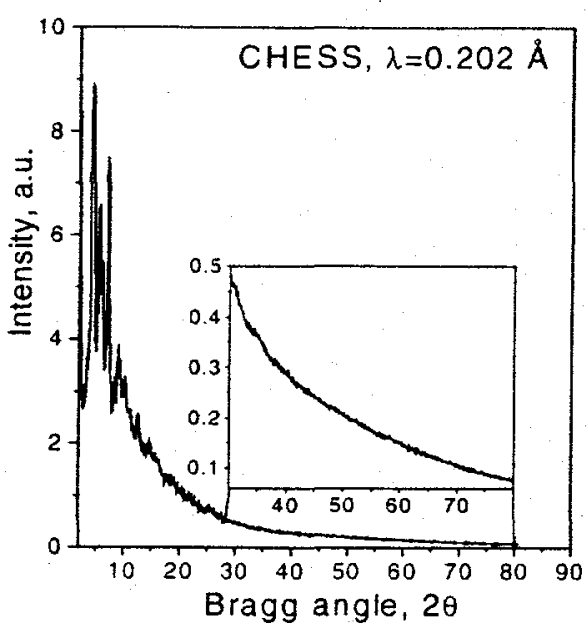

Figure 3. X-ray diffraction spectrum of "restacked" WS.

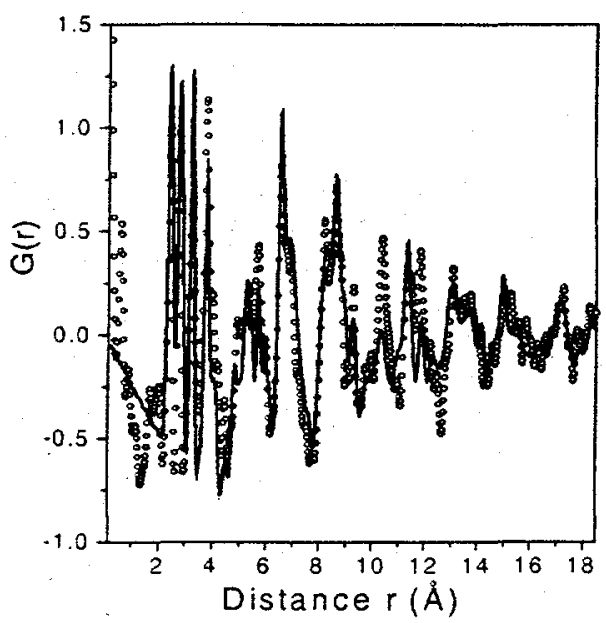

Figure 4. Experimental (symbols) and model (solid line) atomic PDFs of "restacked" WS 2 .

[8 and refs. therein]. The chemistry of these materials is particularly fascinating since the individual $\left(W S_{2}\right)$ layers can be blown and kept apart in water for days. The material can be "restacked" by precipitation, evaporation or centrifugation and "guest species" can be encapsulated between the layers. There has been a lot of confusion about the structure of "restacked" $W S_{2}$. The structure has been proposed to be trigonal TiS type [8]. Recent electron diffraction study, however, showed that the structure (two-dimensional xy-plane) of the single $\left(W S_{2}\right)$ layers is similar to that of the orthorhombic $W T e_{2}$ [9]. In this structure $W$ atoms within a single layer form zig-zag parallel chains via metal-metal bonds. Since no structural information was obtained in the $\mathrm{z}$-direction, and in order to show that the single crystalline specimens probed by the electron diffraction were representative of the bulk we undertook a PDF study with the use of $\mathrm{X}$-rays of energy $61 \mathrm{keV}$. The experiments were carried out at A2 beamline CHESS. The raw X-ray diffraction spectrum obtained is shown in Fig. 3 and the PDF extracted from it - in Fig. 4. As can be seen in Fig. 3 the diffraction spectrum of "restacked" $W S_{2}$ contains a pronounced diffuse scattering component and only few Bragg peaks which renders the data analysis by ordinary techniques, like the Rietveld refinement, almost impossible. The atomic PDF, however, is rich of structure-related features and lends itself to structure determination. It has been found that the experimental PDF can well be fit with a structure based on a monoclinic unit cell with parameters $a=3.2545(5) \AA, b=5.7092 \AA, c=12.3783(5) \AA ; \beta=87.74^{\circ}$ which can be viewed as a distorted derivative of the unit cell of $W T e_{2}$. The result agrees with the electron diffraction study [9] suggesting that the layered structure of untreated $W S_{2}$ does undergo a considerable distortion down to a monoclinic symmetry when the material is subjected to the chemical processing described above. A more detailed account of the present study will be reported elsewhere [10]. 
Calcium aluminosilocate glasses are among the most frequently man-made glasses. That is why they are subjected to extensive studies. It is generally accepted that these glasses are built of Si-O and $A l-O$ polyhedral units linked together by common oxygen atoms. The so linked units form a continuous network with $\mathrm{Ca}$ ions occupying large irregular cavities in it. Oxygens linking two polyhedral units from the network are called "bridging" while those connecting one $A l$ or Si with

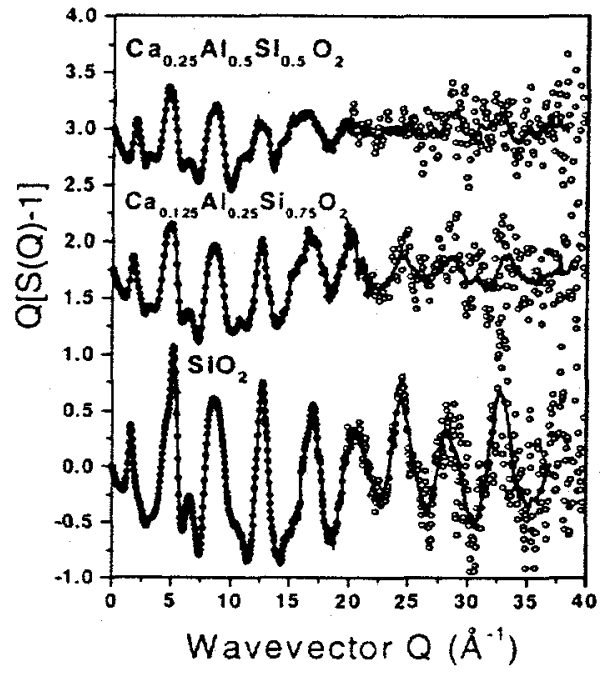

Figure 5. Experimental structure factors for calcium aluminosilicate glasses (dots) together with the optimum smooth line.

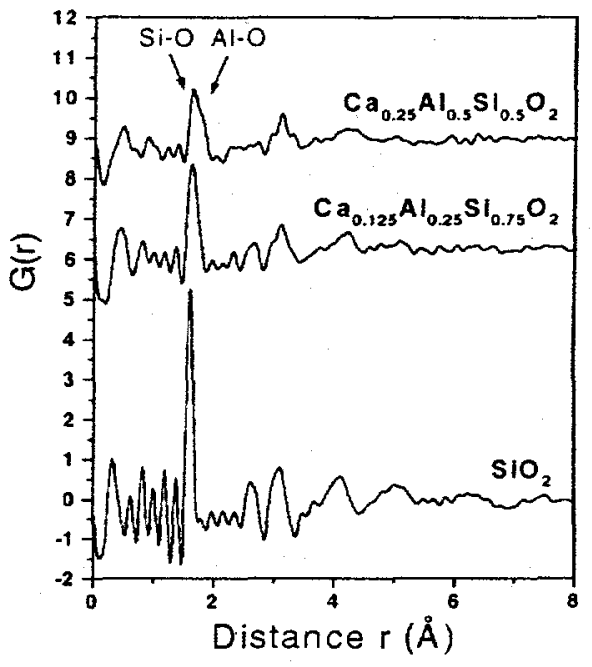

Figure 6. Atomic PDFs, G(r), of calcium aluminosilicate glasses obtained by Fourier transforming the smoothed data of Fig. 5.

$\mathrm{Ca}$ cation - "non-bridging" (NBO). Although NBO are not an integral part of the polyhedral network they play an important role in determining the thermodynamic properties of the glasses. We undertook a PDF study to investigate the nature of Si-O and Al-O polyhedral units and the distribution of NBOs on them. The experiments were carried out at I-BM beamline at the Advanced Photon Source, Argonne with the use of $x$-rays of energy $65 \mathrm{keV}$ [11]. The experimental structure factors are shown in Fig. 5 and the corresponding PDFs - in Fig. 6. Due to the fact that the glassy samples investigated exhibited weak diffuse scattering statistics of the experimental data turned out to be somewhat poor despite the powerful synchrotron source employed. It necessitated some extra smoothing of the data to be carried out, as shown in Fig. 5 . The smoothed $S(Q)$ data show oscillating behaviour. i.e. structure relevant features, up to the maximum $Q$-value of $40 \AA^{-1}$ reached. The corresponding high-resolution $G(r) s$ have a sharp first peak which is obviously composed of two components in $\mathrm{Ca}$ and $A l$ containing glasses. The first component is positioned at $1.61 \AA$ and reflects the presence of well-defined $\mathrm{SiO}_{+}$units. The higher- $r$ component is positioned at approximately $1.75 \AA$ which is the Al-O distance usually found in $\mathrm{AlO}_{+}$tetrahedral units. Thus the present study provides a strong experimental evidence supporting the model picture viewing calcium aluminosilicate glasses as a network of linked $\mathrm{SiO}_{+}$and $\mathrm{AlO}_{+}$tetrahedra. New PDF experiments aimed at improving the statistical accuracy of the data will be carried out soon and the fascinating atomic ordering of $\mathrm{Ca}_{x / 2} \mathrm{Al}_{\mathrm{L}} \mathrm{Si} i_{l_{\mathrm{K}} \mathrm{O}} \mathrm{O}_{2}$ glasses, 
including the distribution of NBOs on the individual $\mathrm{SiO}_{4}$ and $\mathrm{AlO}_{4}$ tetrahedra, revealed in more detail.

\section{CONCLUSIONS}

The combination of intense high-energy sources of $\mathrm{x}$-ray radiation, such as synchrotrons, and the atomic Pair Distribution Function technique offers new opportunities for exploring the structure of materials with higher real-space resolution. It can be fruitfully applied for investigating the atomic arrangement in completely disordered materials like glasses, revealing fine local deviations from an well known average structure, like the case of $\operatorname{In}_{1-x} G a_{x} A s$ semiconductor alloys considered, and even for determining of unknown structures, like the one of "restacked" $W S_{2}$. It is envisaged to be much more frequently employed for materials structure studies with the advent of the new generation synchrotron sources.

\section{ACKNOWLEDGEMENTS:}

Thanks are due to Drs. Th. Proffen, D. Haeffner and Mr. I-K. Jeong for discussions and help with the experiments. The work was supported by DOE grant DE FG02 97ER45651 and by NSF grant CHE 99-03706. S.J.L.B. also acknowledges support from Alfred P. Sloan Foundation. CHESS is operated by NSF through grant DMR97-13242. Advanced Photon Source is supported by DOE under contract W-31-109-Eng-38.

\section{REFERENCES:}

1. H.M. Rietveld, J. Appl. Cryst. 2 (1969) 65.

2. The Rietveld Method edited by R.A. Young, Oxford University Press (1995).

3. T. Egami in Local structure from Diffraction edited by S.J.L. Billinge and M.F. Thorpe, p. 1, New York, Plenum. 1998.

4. Y. Waseda, The structure of non-crystalline materials, New York, McGraw Hill, 1980.

5. V. Petkov, J. Appl. Cryst. 22 (1989) 387.

6. V. Petkov, I-K. Jeong, J. Chung, M.F. Thorpe, S. Kycia and S.J.L. Billinge, Phys. Rev. Lett. 83 (1999) 4089.

7. V. Petkov, [-K. Jeong, F.M-Jacobs, Th. Proffen, J.S.L. Billinge and W. Dmowski, J. Appl. Phys., submitted.

8. R. Chianelli, J. Scanlon and A. Thompson, Mat. Res. Bull. 10 (1975) 1379.

9. J. Heising and M. G. Kanatzidis, J. Am.Chem. Soc. 121 (1999) 638

10. V. Petkov, S.J.L. Billinge, J. Heising and M. G. Kanatzidis, in preparation

11. V. Petkov, I-K. Jeong. M. Gutmann, P.F. Peterson, S.J.L. Billinge, S. Shastri and

B. Himmel, Advanced Photon Source User Activity Report www.aps.anl.gov/xfd/communicator/user2000/sri-cat.html 